\title{
Étude du rayonnement U.V. du radical SiO appliqué à des mesures d'absorption auto-accordée
}

\author{
F. Coursimault, O. Motret et J.-M. Pouvesle \\ GREMI, UMR 6606 du CNRS, BP. 6744, 45067 Orléans cedex 2, France
}

\begin{abstract}
Résumé : Cette étude s'inscrit dans le cadre de la mise au point d'une technique de mesure de densité absolue de la molécule $\mathrm{SiO}$, cette dernière intervenant dans la physico-chimie des traitements de film polymère par plasma. Le diagnostic développé est basé sur l'absorption d'un rayonnement U.V. produit par la même molécule que celle à sonder. Nous présentons ici les résultats concernant la mise au point de la source U.V. permettant de produire la fluorescence de SiO. Plusieurs bandes vibrationnelles ainsi que la structure rotationnelle de la transition $\mathrm{A}-\mathrm{X}$ ont pu être étudiées dans différents gaz porteurs.
\end{abstract}

\section{INTRODUCTION}

L'amélioration des propriétés de surface de films polymères (mouillabilité, adhérence) a des débouchés industriels importants. Parmi les différentes techniques possibles, les procédés faisant appel à l'utilisation de plasmas froids (décharge couronne, Décharge à Barrière Diélectrique) semblent bien adaptés. Cependant, la complexité de la physico-chimie de ces milieux nécessite une meilleure connaissance des processus intervenant dans le volume gazeux au niveau du réacteur afin d'optimiser le procédé. C'est dans ce cadre que s'inscrivent nos travaux. Ils ont pour but de développer un diagnostic optique souple permettant de mesurer des concentrations absolues de composés moléculaires, notamment SiO, intervenant dans les processus de dépôt. La technique employée est l'Absorption d'un rayonnement U.V Extérieur Auto-accordé (technique AREA), déjà utilisée pour la mesure des radicaux OH [1] dans des plasmas de même nature. Les travaux présentés ici concernent l'étude et le développement de la source lumineuse.

\section{RESULTATS}

Le rayonnement lumineux est créé à partir d'une $\mathrm{DBD}$ impulsionnelle dans un mélange atmosphérique contenant du silane, une espèce oxydante $\left(\mathrm{N}_{2} \mathrm{O}, \mathrm{O}_{2}, \ldots\right)$ et un gaz porteur. Deux types de gaz porteurs ont été utilisés l'azote et l'argon. Les mesures d'absorption se font entre un niveau électronique excité et le fondamental, notre choix s'est donc porté sur la transition A-X, s'étendant de 216.35 à $292.53 \mathrm{~nm}$. C'est cette transition qui fait l'objet de cette étude en émission.

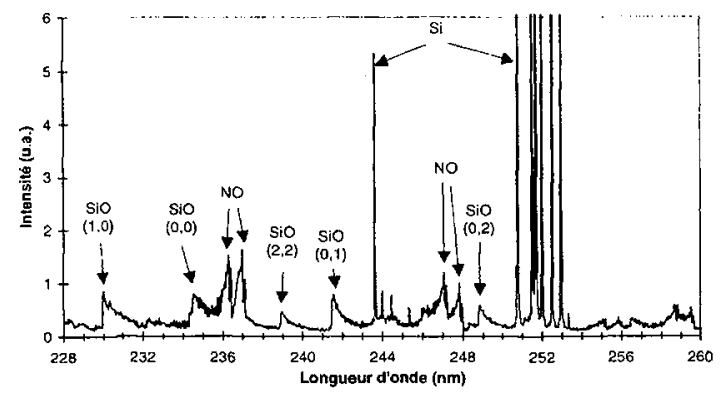

Figure 1. Spectre d'émission réalisé dans un mélange $\mathrm{N}_{2}, \mathrm{SiH}_{4}, \mathrm{~N}_{2} \mathrm{O}(98 \%, 0.3 \%, 1.5 \%)$

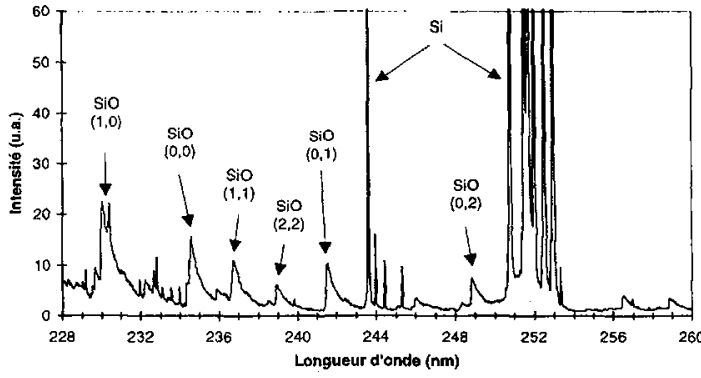

Figure 2. Spectre d'émission réalisé pour un mélange $\mathrm{Ar}, \mathrm{SiH}_{4}, \mathrm{~N}_{2} \mathrm{O},(98.55 \%, 0.38 \%, 1.07 \%)$ 
Les premiers résultats dans l'azote (figure 1) ont montré la présence de plusieurs bandes vibrationnelles de $\mathrm{SiO}((1,0),(0,0),(2,2),(0,1),(0,2))$, ainsi qu'un nombre important d'autres émissions tel que le système $\gamma$ de NO et les émissions atomiques du silicium. La présence de fluorescence parasite est un inconvénient pour l'utilisation du rayonnement de SiO. Pour résoudre ce problème, le gaz porteur azote a été remplacé par l'argon. Les spectres ainsi obtenus (figure 2) sont ainsi exempts des bandes de NO. Une autre bande moléculaire de $\mathrm{SiO}(1,1)$, jusqu'alors noyée dans l'émission de NO, a pu être isolée à $236.57 \mathrm{~nm}$. De façon générale le gaz porteur ne semble pas influencer considérablement la chimie de formation de $\mathrm{SiO}$, basée semble-t-il sur les radicaux $\mathrm{OH}$ et $\mathrm{O}$ [2]. Ce mécanisme réactionnel conduit quel que soit le gaz porteur à la formation de poudre de silice très gênante pour les applications basées sur l'absorption. Ce phénomène est l'un des problèmes majeurs dans la mise au point de ce diagnostic.

La structure rotationnelle de la bande vibrationnelle $(1,0)$ de la transition $\mathrm{A}-\mathrm{X}$ de $\mathrm{SiO}$ dans l'argon est représentée figure 3.

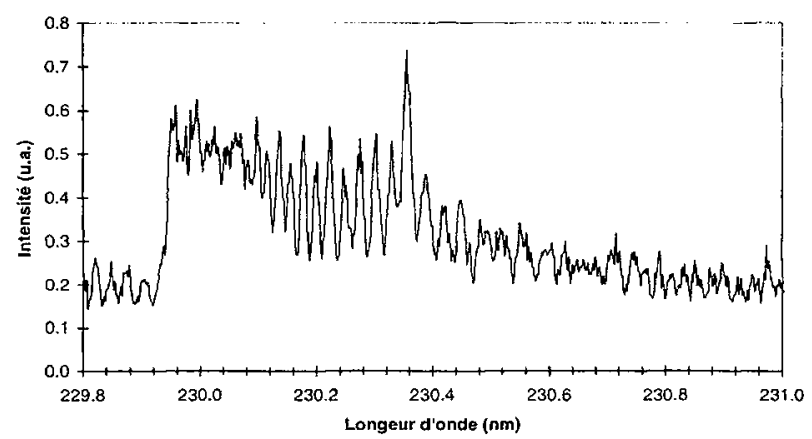

Figure 3. Spectre rotationnel de $\mathrm{SiO}$, bande vibrationnelle (1,O). $\mathrm{Ar}, \mathrm{SiH}_{4}, \mathrm{~N}_{2} \mathrm{O}(98.55 \%, 0.38 \%, 1.07 \%)$

Nos résultats montrent une tête de bande peu intense. Ce phénomène est confirmé par d'autres travaux [3] réalisés par Hyne par LIF dans des flammes à pression atmosphérique. La structure très resserrée de ces raies rotationnelles ne semble pas permettre avec le dispositif spectroscopique dont nous disposons de pouvoir isoler une seule raie pour effectuer le travail d'absorption comme cela a pu être le cas pour $\mathrm{OH}$ [1]. Les mesures d'absorption seront donc réalisées sur la totalité d'une transition vibrationnelle.

\section{CONCLUSION}

Les premières investigations sur le rayonnement de la molécule SiO impliquent déjà des premiers choix pour la mise au point de la technique AREA. Tout d'abord, il sera préférable d'utiliser un mélange à base d'argon pour la création de la source afin de s'affranchir au maximum d'émission parasite de composés moléculaires et atomiques. Il sera possible d'obtenir plusieurs estimations de la concentration de $\mathrm{SiO}(\mathrm{X})$ à partir des différentes bandes vibrationnelles observées. Mais le problème majeur reste la formation de poudre qui en s'accumulant sur les électrodes, dégrade les performances du système au cours du temps.

\section{Remerciements}

Ce travail a bénéficié du soutien financier du groupe l'Air Liquide.

\section{Références}

[1] C. Hibert, I. Gaurand, O. Motret, and J.M. Pouvesle, "[OH(X)] measurements by resonnant absorption spectroscopy in a pulsed dielectric barrier discharge $»$, J. Appl. Phys. Vol. 85, N¹0, (1999), 7070-7075.

[2] N. Gherardi, «Physico-chimie et régimes des décharges contrôlées par barrière diélectrique en mélanges azote - gaz oxydant - silane. Application a l'activation de surface polymères et aux dépôts. », thèse de l'université Paul Sabatier, Toulouse, 1999

[3] A. J. Hynes, «Laser-induced fluorescence of silicon monoxyde in a glow discharge and an atmosphric pressure flame », Chem, Phys. Letters, Vol. 181, (1991), P 237. 